[Highlight]

\title{
一种调控催化剂性能的新要素: 金属一载体的晶面协同效应
}

\author{
张铁锐 \\ (中国科学院理化技术研究所, 北京 100190)
}

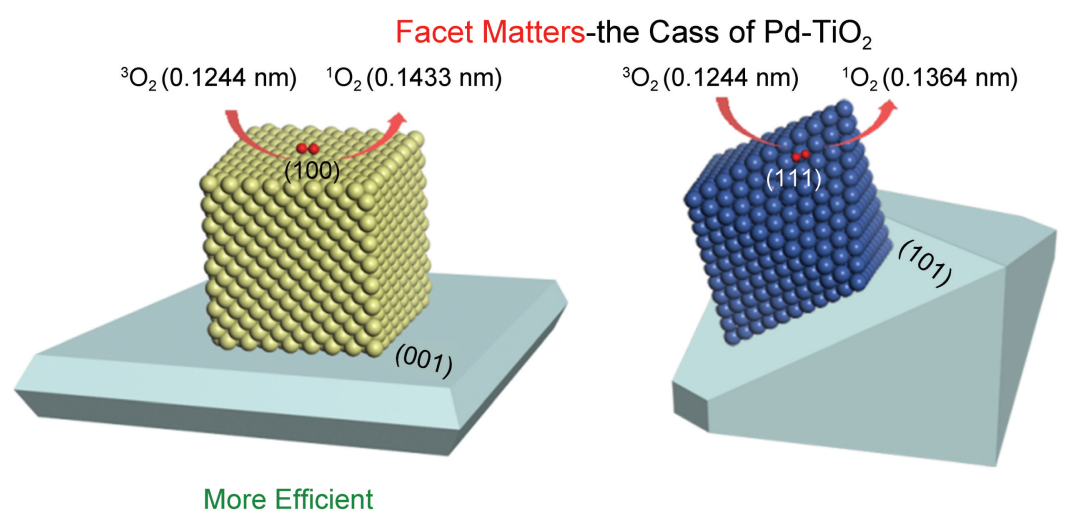

催化工业是现代社会发展的重要基石之一。 氧化物负载金属颗粒的多相催化体系在整个催化 工业中具有重要的地位。在催化科学发展的初 期，一般认为氧化物载体只起到简单的支撑、分 散活性组分的作用, 不会影响到金属催化剂的性 能。但是随着研究的不断深入, 人们逐渐意识到 合适的载体能极大地增强负载催化剂的性能。特 别是 Tauster 等 ${ }^{1}$ 于 1978 年提出 “金属一载体强相互 作用” (strong metal-support interaction) 的概念之 后, 金属活性成分与载体之间的相互作用引起了 越来越多的关注。

随着纳米科技的兴起, 人们对各类具有特定 曝露晶面的纳米晶体的制备及其催化应用也做了 深入的研究, 并取得了一系列研究成果。前人的 大量研究表明, 改变纳米粒子的形貌, 即调控其 曝露的晶面, 是增强纳米材料催化性能的有效途 径; 这是因为催化过程都发生在材料的表面上, 晶面的差异会影响其反应速率。例如, 在氧的催 化活化反应中, 曝露(100)晶面的 Pd 立方体颗粒的 催化性能远远高于曝露(111) 晶面的 Pd八面体颗 粒 ${ }^{2,3}$ 。同样, 在 $\mathrm{Au} 、 \mathrm{Ag} 、 \mathrm{Pt}$ 等贵金属材料以及 $\mathrm{TiO}_{2} 、 \mathrm{CdS} 、 \mathrm{BiOBr} 、 \mathrm{Ag}_{3} \mathrm{PO}_{4}$ 等半导体材料中, 不 同晶面也能够极大地影响纳米材料的催化活性 ${ }^{4}$ 。 在目前已知的金属-半导体复合体系中, 大家基本 上都着眼于寻找合适的组分或者单独调控某一种
组分的晶面, 以期实现更高的催化活性、更高的 催化选择性 ${ }^{5}$, 较少有人关注载体晶面和金属晶面 之间有无相互作用。

最近, 苏州大学功能纳米与软物质研究院张 桥教授与林海平博士合作, 以 $\mathrm{Pd}-\mathrm{TiO}_{2}$ 复合材料为 目标体系, 分别从理论计算和实验角度对载体晶 面与金属晶面之间的相互作用进行了研究 ${ }^{6}$ 。该团 队选择了 $\operatorname{Pd}(100)$ 和 $\operatorname{Pd}(111)$ 晶面作为典型的金属晶 面, 并选择了 $\mathrm{TiO}_{2}(001)$ 和 $\mathrm{TiO}_{2}(101)$ 晶面作为典型 的半导体晶面。首先利用密度泛函理论(DFT)研究 不同的 $\mathrm{TiO}_{2}$ 晶面与不同 $\mathrm{Pd}$ 晶面组合上的局域态密 度(LDOS)和分子氧的吸附状态。LDOS 模拟结果 表明, 在 $\mathrm{TiO}_{2}(001)$ 晶面上的 $\mathrm{Pd}$ 晶体比负载于 $\mathrm{TiO}_{2}$ (101)表面的 $\mathrm{Pd}$ 晶体更为活泼; 另一方面, 在同一 $\mathrm{TiO}_{2}$ 的晶面上, $\mathrm{Pd}(100)$ 则比 $\mathrm{Pd}(111)$ 表现出更高的 活性。分子氧的活化模拟结果也表明, 单线态分 子氧 $\left({ }^{1} \mathrm{O}_{2}\right)$ 键长顺序为 $\mathrm{Pd}(100)-\mathrm{TiO}_{2}(001)>\mathrm{Pd}(100)-$ $\mathrm{TiO}_{2}(101)>\mathrm{Pd}(111)-\mathrm{TiO}_{2}(001)>\mathrm{Pd}(111)-\mathrm{TiO}_{2}(101)$, 表明 $\mathrm{Pd}(100)-\mathrm{TiO}_{2}(001)$ 的复合体系具有最高的催化 活性。在电子自旋共振(ESR)表征分子氧活化性能 与光催化氧化葡萄糖的反应中, 与理论预测结果 一致, 复合体系的催化性能与 $\mathrm{Pd}$ 晶面及 $\mathrm{TiO}_{2}$ 载体 的晶面类型密切相关, 且与 $\mathrm{TiO}_{2}(001)$ 晶面比例呈 现明显的正相关关系。相关工作近期发表在 Nano Letters 上, 题目为 The synergy between metal facet 
and oxide support facet for enhanced catalytic performance: the case of $\mathrm{Pd}-\mathrm{TiO}_{2}$ 。这一研究结果提出了一 种调控催化剂性能的新要素: 金属与载体的晶面 协同效应, 从而为设计具有更高催化性能的催化 材料提供了一条新的思路。

\section{References}

(1) Tauster, S.; Fung, S.; Garten, R. J. Am. Chem. Soc. 1978, 100 (1), 170. doi: $10.1021 / \mathrm{ja} 00469 \mathrm{a} 029$

(2) Jin, M.; Zhang, H.; Xie, Z.; Xia, Y. Energy Environ. Sci. 2012, 5 (4), 6352. doi: 10.1039/c2ee02866b
(3) Long, R.; Mao, K.; Ye, X.; Yan, W.; Huang, Y.; Wang, J.; Fu, Y.; Wang, X.; Wu, X.; Xie, Y.; Xiong, Y. J. Am. Chem. Soc. 2013, 135 (8), 3200. doi: 10.1021/ja311739v

(4) Chiu, C. Y.; Chung, P. J.; Lao, K. U.; Liao, C. W.; Huang, M. H. J. Phys. Chem. C 2012, 116 (44), 23757. doi: 10.1021/jp307768h

(5) Long, R.; Mao, K.; Gong, M.; Zhou, S.; Hu, J.; Zhi, M.; You, Y.; Bai, S.; Jiang, J.; Zhang, Q.; Wu, X.; Xiong, Y. Angew. Chem. Int. Ed. 2014, 53 (12), 3205. doi: 10.1002/anie. 201309660

(6) Cao, M. H.; Tang, Z. Y.; Liu, Q. P.; Xu, Y.; Chen, M.; Lin, H. P.; Li, Y. Y.; Gross, E.; Zhang, Q. Nano Lett. 2016, 16 (8), 5298. doi: 10.1021/acs.nanolett.6b02662 